\title{
Compton Thick AGN
}

\author{
N. A. Levenson \\ Gemini Observatory, Casilla 603, La Serena, Chile \\ email: nlevenson@gemini.edu
}

\begin{abstract}
Compton thick active galactic nuclei (AGN), which are obscured by column density $N_{H}>1.5 \times 10^{24} \mathrm{~cm}^{-2}$, can be difficult to identify. They are certainly cosmically significant, both in producing the observed cosmic X-ray background, and in providing a location where black hole growth is hidden from view. Here I review some recent results from surveys that provide indications of Compton thick AGN, considering X-ray, radio, and infrared selection techniques. I also offer a caution against using mid-infrared silicate features to measure line-ofsight obscuration to active galactic nuclei. Instead, these features better indicate the geometric distribution of dust that the central engine heats. I conclude that the outstanding problem of Compton thick AGN is not the cases where the obscuration is directly associated with the environment of the active nucleus itself, even in the most obscured examples. Instead, we still risk missing the completely buried AGN, which are obscured by large amounts of gas and dust over large solid angles. The solution to finding Compton thick AGN may be to begin the search based on infrared emission and star formation, and then select for nuclear activity.
\end{abstract}

Keywords. AGN, obscuration, dust

\section{Introduction to Compton Thick AGN}

At first blush, completing surveys of active galactic nuclei (AGN) should be straightforward. After all, these sources are extremely luminous across a broad range of wavelengths, and the active nuclei are often more powerful than the entire host galaxy in which they reside. The complication, however, is obscuration. The standard "unified" model of AGN provides some optically and geometrically thick material that hides the powerful central engine from some lines of sight, which accounts for a wide variety of observational properties in terms of geometry and viewing perspective. The situation is further challenging in the case of Compton thick AGN, those that are obscured by column density $N_{H}>1.5 \times 10^{24} \mathrm{~cm}^{-2}$. At these high column densities, the obscuring region absorbs even $\mathrm{X}$-rays, in addition to being optically thick at visible through infrared wavelengths. The soft X-rays, those having energy less than $10 \mathrm{keV}$, are nearly completely absorbed.

The Compton thick AGN are important both in consideration of the cosmic X-ray background (XRB) and to the general subject of observing and quantifying black hole evolution. The XRB is not a true "background." Rather, it is composed of the net contribution of discrete sources, predominantly AGN. The observed spectral peak of the $\mathrm{XRB}$ is around $20 \mathrm{keV}$, and addition of a significant population of Compton thick AGN (10\% by number of greater) can reproduce this spectral shape. Figure 1 shows measurements of the XRB fit by a variety of models. Note that many different models, having very different assumptions about the number and cosmic distribution of Compton thick AGN, all reproduce the observed spectrum. The XRB is a sign that Compton thick AGN are cosmically important, but it leaves the details of their number and distribution to be determined through other methods. 


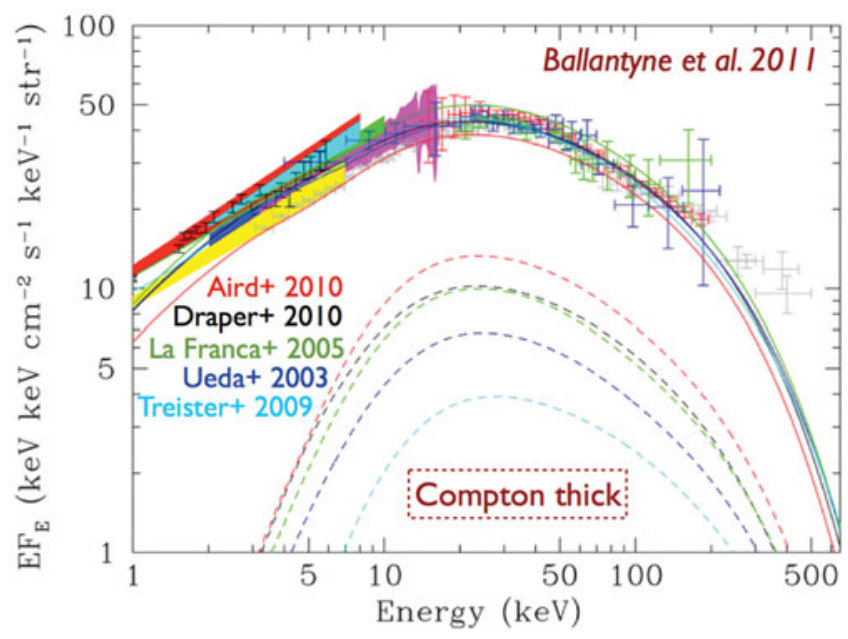

Figure 1. Observations of the XRB (points) and models that describe it (lines). Many different prescriptions for Compton thick AGN (dashed lines) can reproduce the observed XRB. (From Ballantyne et al. 2011.)

The second key reason Compton thick AGN are important is in the context of galaxy evolution. We want a complete census (and even detailed demographics) of black holes to quantify their growth in galaxies and their relationship to their host galaxies. Missing direct observations of some black holes, especially those that may be buried in dusty merging galaxies, means that we are missing a potentially critical phase that sets the stage for further co-evolution of black holes and galaxies.

X-ray observations are particularly important to the study and identification of AGN, especially those that are Compton thick. AGN are bright X-ray point sources, and there is little confusion for other sources when the $\mathrm{X}$-ray luminosity is large, (e.g., $L_{X}>$ $10^{41} \mathrm{erg} \mathrm{s}^{-1}$ ). X-rays measurements are especially useful because they can be scaled to estimate the AGN bolometric luminosity. Several of the techniques discussed below will rely on this point, and evidence of obscuration is typically based on the difference between expected and observed X-ray flux.

In addition to weak soft emission, X-rays offer two further spectral signatures of Compton thick obscuration: a flat X-ray spectrum (in contrast to the sloped power law that the intrinsic and unobscured AGN display), and a large equivalent width $\mathrm{Fe} \mathrm{K} \alpha$ line (Figure 2). This fluorescent emission line is produced in the obscuring medium, and equivalent width greater than $1 \mathrm{keV}$ is characteristic of Compton thick AGN. In addition, this line luminosity is roughly related to intrinsic luminosity, with $L_{X} \approx 500 L_{F e}$ (Levenson et al. 2002), providing another indication of intrinsic luminosity when the continuum cannot be observed directly.

\section{Results}

I concentrate here on general conclusions about the frequency and distribution of Compton thick AGN. More targeted surveys specifically in search of these sources can effectively find additional individual cases; see Vignali (this volume, and references therein) for some examples of these techniques and other perspectives on Compton thick AGN.

\subsection{X-ray Surveys}

The Chandra Deep Field South currently provides the deepest X-ray observations at energies up to $10 \mathrm{keV}$, with total exposure of $4 \mathrm{Ms}$. Over 700 sources are detected (Xue et al. 

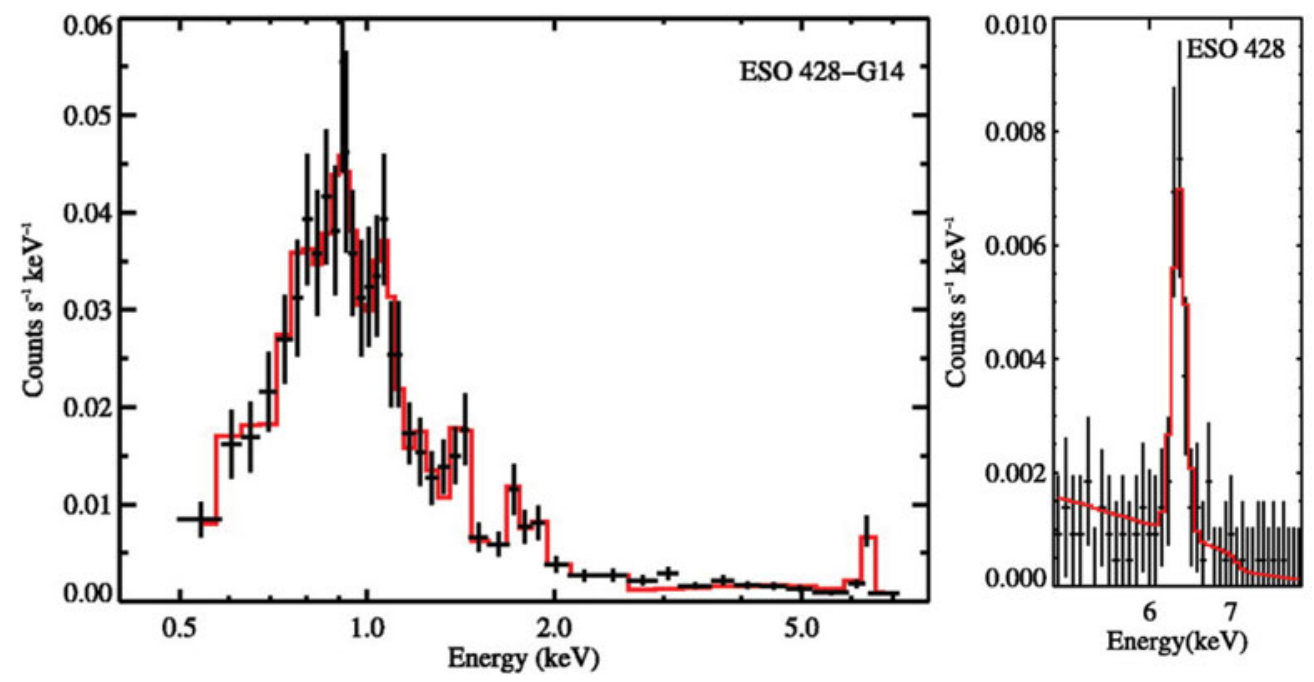

Figure 2. (left) The Compton thick AGN ESO 428-G14 exhibits a flat and weak X-ray spectrum at energies above $2 \mathrm{keV}$. The soft X-ray emission does not show the intrinsic central engine directly; rather, it is due to photoionization, analogous to the optical narrow line region. The $\mathrm{Fe} \mathrm{K} \alpha$ line is apparent, and more obvious in the close-up view (right) at a rest wavelength of $6.4 \mathrm{keV}$. (From Levenson et al. 2006.)

2011), most of which have multi-wavelength counterparts to identify them as galaxies. Brightman \& Ueda (2012) present a recent analysis of the Compton thick fraction in this field. They concentrate on the brighter sources, which provide higher quality X-ray spectral characterization. They conclude that $10 \%$ of the AGN are Compton thick (including unconfirmed candidate sources). Considering observational biases, Brightman \& Ueda (2012) predict that $20 \%$ of local AGN are Compton thick, with up to $40 \%$ at $z>1$. This work is consistent with earlier results, including the general trend of more heavily obscured AGN at higher redshift (e.g., La Franca et al. 2005).

The Burst Alert Telescope (BAT) on the Swift satellite provides an all-sky survey at energies 14-195 keV, to a flux limit of $10^{-11} \mathrm{erg} \mathrm{s}^{-1} \mathrm{~cm}^{-2}$ (Baumgartner et al. 2013). Five per cent of the observed AGN are Compton thick, and correcting for selection effects indicates an actual Compton thick fraction of 20\% (Burlon et al. 2011; Figure 3). Even at these higher energies, the intrinsic radiation of the central engine does not fully escape; only $30 \%$ of the flux of the AGN is visible from these Compton thick examples.

The Nuclear Spectroscopic Telescope Array (NuSTAR) provides measurements in the 6-79 keV range, with the advance of a focusing telescope. The initial results relevant here are based on the first ten identified sources that were serendipitously detected in deep, pointed observations of known targets of interest (Alexander et al. 2013). These sources have fluxes around $10^{-13} \mathrm{erg} \mathrm{s}^{-1} \mathrm{~cm}^{-2}$ and are typically ten times more luminous than those of the BAT survey. None of these serendipitous sources are Compton thick, and correcting for observational limitations and small numbers, the authors set an upper limit on the Compton thick fraction of around $33 \%$.

\subsection{Radio Selection}

Sample selection at radio wavelengths has the advantage of being unbiased for orientation, but is a potential disadvantage because most AGN are not radio loud. With only about $10 \%$ of AGN being radio loud, survey results may not reveal the fundamental nature of most active galaxies. Recent work by Wilkes et al. (2013) uses a complete, flux-limited 


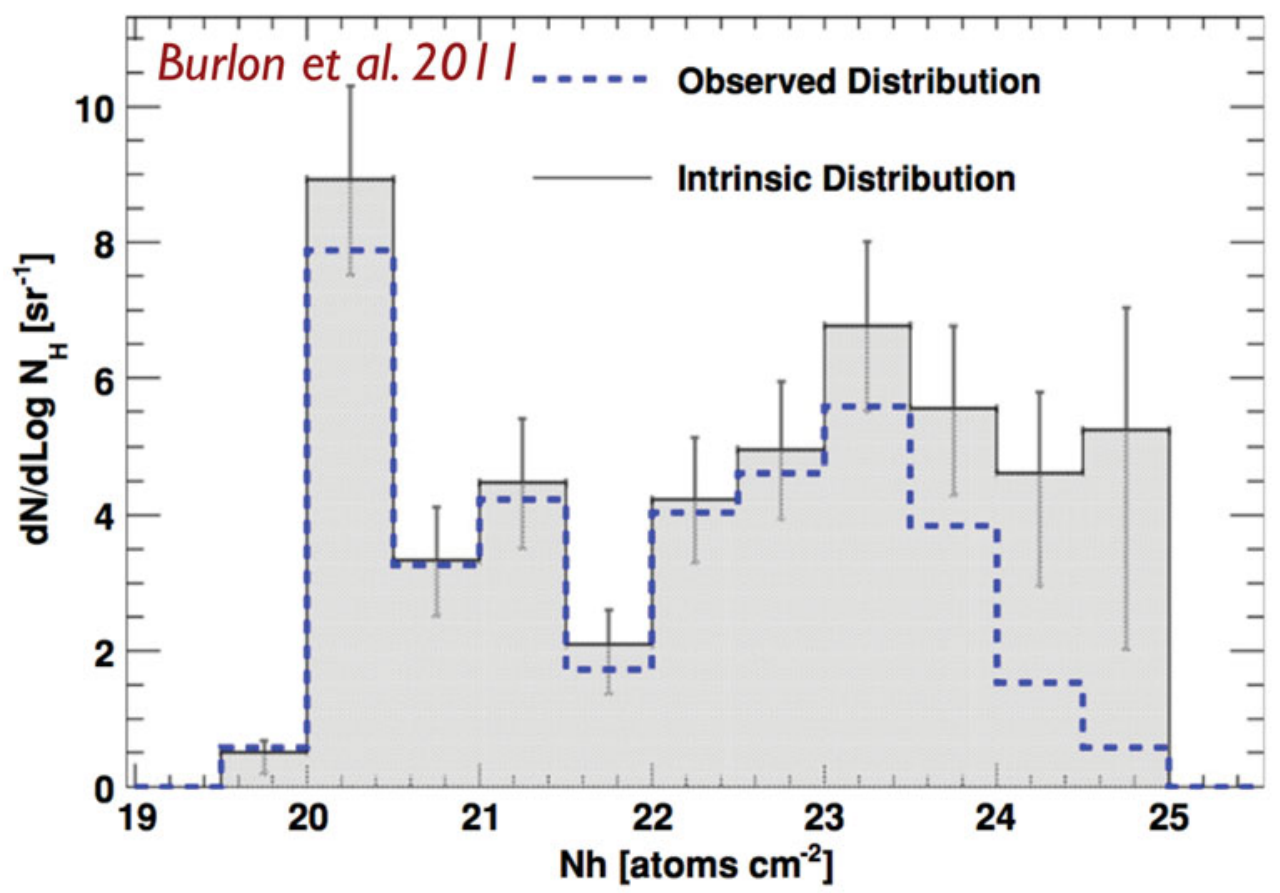

Figure 3. Column density distribution of Swift BAT AGN survey. Even observed at energy above $14 \mathrm{keV}$, not all AGN are detected directly. Correcting for selection effects results in a significantly larger fraction of Compton thick AGN than are observed. (From Burlon et al. 2011.)

sample of 38 known AGN at redshift $1<z<2$, finding a Compton thick fraction of $20 \%$.

Measurements of "radio excess" provide a different selection. The underlying expectation is that star formation produces correlated radio and far-infrared emission. Excess radio flux beyond this correlation is then attributed to an AGN. The determination of absorption relies on a comparison between observed and predicted X-ray flux, with the most deficient sources in observed X-rays being Compton thick candidates. In recent analysis of star-forming galaxies in the Great Observatories Origins Deep Survey (GOODS)-North field, Del Moro et al. (2013) classify $11 \%$ of the $z<3$ galaxies that have radio and far-infrared detections as radio excess AGN candidates, and they suggest that up to $43 \%$ of these radio excess galaxies may be Compton thick.

\subsection{Infrared Approaches}

Mid-infrared emission provides another indicator of intrinsic bolometric luminosity. The observed core mid-infrared luminosity is explicitly correlated with intrinsic X-ray emission (Horst et al. 2006; Gandhi et al. 2009; Levenson et al. 2009; Asmus et al. 2013). Nearby dusty material reprocesses radiation of the central engine, so it emerges at longer wavelengths, and the infrared emission is nearly isotropic. Infrared measurements on small spatial scales are particularly valuable to isolate the AGN, avoiding confusion from stellar contributions. Similar to other techniques described above, mid-infrared selection can be used to identify Compton thick AGN, taking an observed X-ray deficit (relative to expectations) as a sign of obscuration. 
Infrared colors effectively select luminous AGN, although they can miss many or most of the low-luminosity examples. The color selection aims to distinguish the hotter dust of AGN from the cooler spectra of individual stars and inactive galaxies. Thus, the AGN appear "redder" than inactive galaxies these infrared selections. Established work uses colors defined by the Spitzer Infrared Array Camera (IRAC) passbands (Stern et al. 2005; Lacy et al. 2007), where the AGN locus is a wedge in color-color plots. Some more recent studies are based on observations from the Wide-field Infrared Survey Explorer (WISE), which offers all-sky but shallower coverage (Stern et al. 2012; Mateos et al. 2013). A further constraint can be to require explicitly an infrared spectral power law, to avoid contamination from dusty star-forming galaxies, which can be a problem especially at high redshift (Donley et al. 2012). In order to determine the obscuration, comparison with X-ray data is required. The fraction of obscured AGN of some type is typically high using infrared selection (around half), revealing the bias of basing AGN selection on non-isotropic emission. However, these studies do not generally show specific evidence for large numbers of Compton thick AGN. Overall, these infrared color techniques are reliable for selecting AGN (providing a high success rate of confirmed AGN), but not necessarily yielding complete samples, especially at low luminosity.

\section{A Caution}

The silicate dust features at mid-infrared wavelengths (around 10 and $18 \mu \mathrm{m}$ ) have been used to assess the column density to AGN and therefore identify Compton thick examples, but in fact this method provides an inaccurate measurement. The fundamental problem is that the silicate features in absorption do not indicate the actual line-of-sight optical depth but only the apparent optical depth, $\tau_{a p p}$. Figure 4 shows characteristic examples of several different classes of galaxies (Levenson et al. 2007). The Seyfert 2, NGC 5643, is instructive here. The silicate optical depth can be measured relative to the continuum, $\tau_{a p p}=\ln \left(F_{\text {obs }} / F_{\text {cont }}\right)$. The result is $\tau_{a p p}=0.4$, which corresponds to $N_{H}=2 \times 10^{22} \mathrm{~cm}^{-2}$. The obvious challenge is that NGC 5643 is Compton thick, blocked by a column that is orders of magnitude greater!

The calculation for optical depth (as the logarithmic ratio of observed feature to continuum) is correct in the case of a cold, foreground screen. It is generally inapplicable at the centers of active galaxies because the dust emits as well as absorbs radiation at these feature wavelengths. In addition, a cold foreground screen model cannot also be the source of the significant mid-infrared emission that is observed in AGN and correlated with the X-ray radiation. The characteristic silicate features in optically-identified AGN are weak. In type 1 AGN, where spectrally broad optical lines are observed, the silicate generally appears weakly in emission, and in type 2 AGN, which lack broad optical lines, the silicate features typically show weak absorption. A clumpy or inhomogeneous "torus" model on parsec scales around the central engine accounts for these general observations, and specific configurations quantitatively illustrate large column density and weak midinfrared features, even when the line-of-sight column density is large (i.e., corresponding to optically thick silicate feature; Nenkova et al. 2008).

The mid-infrared silicate features are generally useful to reveal the geometry of the dust distribution at the centers of galaxies. (See Alonso-Herrero, this volume, for a recent application of these methods.) Deep absorption is common in ultraluminous infrared galaxies (ULIRGs) that are not optically identified as AGN. These observations are described well by smooth full-covering shells of material (Sirocky et al. 2008), not the clumpy distributions that produce weak features. The ULIRGs are potentially important to the question of Compton thick AGN. They are not obviously AGN according to many 


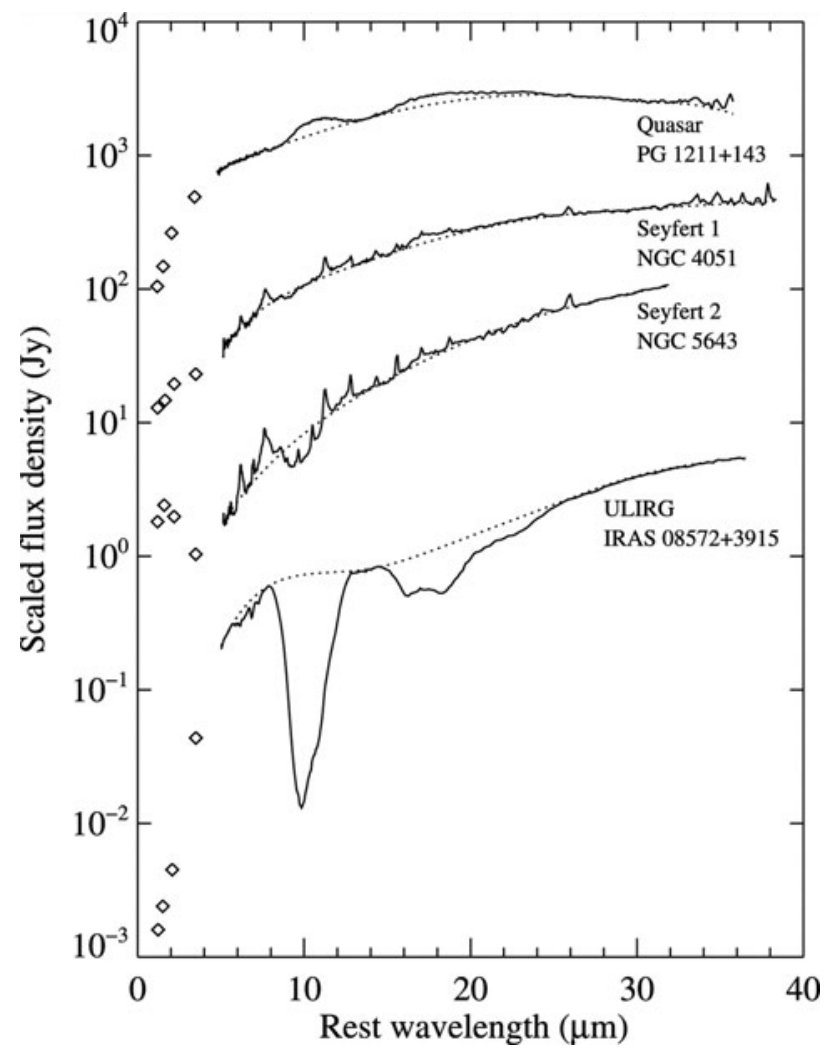

Figure 4. Silicate features are evident in some mid-infrared spectra, although they do not provide an accurate measurement of the line-of-sight optical depth. These examples of various classes of active galaxies show characteristic silicates around 10 and $18 \mu \mathrm{m}$ compared with the fitted continua (dotted lines). These features are very weak in all of the optically-identified AGN, in both emission (quasar and Seyfert 1 classes) and absorption (Seyfert 2), even though this last example is a Compton thick AGN. Deep silicate absorption is common only in ULIRGs that are not optically identified as AGN. (From Levenson et al. 2007.)

diagnostic tests, yet they certainly contain a deeply buried and powerful energy source. Moreover, they may generally represent a typical phase of galaxy evolution that promotes black hole growth by making material for accretion available in the nuclear region.

\section{Conclusions}

Extremely deep and high-energy X-ray surveys find most "ordinary" Compton thick AGN, at least in sufficient numbers to account for most of the XRB. By "ordinary" I mean those cases where the absorption is directly associated with the typical environment of the active nucleus itself: accretion and outflow phenomena and the dusty torus, which are confined to physical scales of parsecs and smaller. The open questions that remain in these cases are the redshift distribution and luminosity dependence. These are details at some level, as many different prescriptions can account for the observations -including the spectrum of the X-ray background - though important to describe the evolution of AGN.

The deeper outstanding problem is the completely obscured AGN, those that are entirely obscured by large amounts of gas and dust and over large covering fractions (large solid angles). These examples will not necessarily exhibit other signs of activity to be identified as AGN at all. These completely obscured nuclei especially arise in galaxies dominated by star formation. Thus, accounting for their number and properties is a significant problem in the context of galaxy evolution and black hole growth. This star formation phase is also (potentially) when the black hole is growing, so these represent the hidden black holes we especially need to identify and measure. 
The model of Sanders et al. (1988) provides the context: gas-rich galaxies merge, then go through a phase of being a ULIRG, and only later does the buried AGN clear its surroundings to reveal itself as an "ordinary" active nucleus. The infrared-luminous phase deserves further attention in the study of AGN. With the existing deep X-ray observations and soon the analysis of the latest hard X-ray surveys, the results that start from AGN identification and then measure for obscuration are likely nearly complete in their yield of Compton thick AGN (after including their accounts for selection effects). The area for concentration in future surveys is the star-forming galaxies. Among these infrared luminous examples, additional Compton thick AGN may be hidden.

NAL is supported by the Gemini Observatory, which is operated by the Association of Universities for Research in Astronomy, Inc., on behalf of the international Gemini partnership of Argentina, Australia, Brazil, Canada, Chile, and the United States of America.

\section{References}

Alexander, D. M., et al. 2013, ApJ, 773, 125

Asmus, D., Gandhi, P., Hönig, S. F., \& Smette, A. 2013, arXiv:1310.2770

Ballantyne, D. R., Draper, A. R., Madsen, K. K., Rigby, J. R., \& Treister, E. 2011, ApJ, 736, 56

Baumgartner, W. H., Tueller, J., Markwardt, C. B., Skinner, G. K., Barthelmy, S., Mushotzky, R. F., Evans, P. A., \& Gehrels, N. 2013, ApJS, 207, 19

Brightman, M. \& Ueda, Y. 2012, MNRAS, 423, 702

Burlon, D., Ajello, M., Greiner, J., Comastri, A., Merloni, A., \& Gehrels, N. 2011, ApJ, 728, 58

Del Moro, A., et al. 2013, A $\mho A, 549$, A59

Donley, J. L., et al. 2012, ApJ, 748, 142

Gandhi, P., Horst, H., Smette, A., Hönig, S., Comastri, A., Gilli, R., Vignali, C., \& Duschl, W. 2009, AESA, 502, 457

Horst, H., Smette, A., Gandhi, P., \& Duschl, W. J. 2006, A\&A, 457, L17

La Franca, F., et al. 2005, ApJ, 635, 864

Lacy, M., Petric, A. O., Sajina, A., Canalizo, G., Storrie-Lombardi, L. J., Armus, L., Fadda, D., \& Marleau, F. R. 2007, AJ, 133, 186

Levenson, N. A., Heckman, T. M., Krolik, J. H., \& Weaver, K. A., Życki, P. T. 2006, ApJ, 648, 111

Levenson, N. A., Krolik, J. H., Życki, P. T., Heckman, T. M., Weaver, K. A., \& Awaki, H., Terashima Y. 2002, ApJ, 573, L81

Levenson, N. A., Radomski, J. T., Packham, C., Mason, R. E., Schaefer, J. J., \& Telesco, C. M. 2009, ApJ, 703, 390

Levenson, N. A., Sirocky, M. M., Hao, L., Spoon, H. W. W., Marshall, J. A., Elitzur, M., \& Houck, J. R. 2007, ApJ, 654, L45

Mateos, S., Alonso-Herrero, A., Carrera, F. J., Blain, A., Severgnini, P., Caccianiga, A., \& Ruiz, A. 2013, MNRAS, 434, 941

Nenkova, M., Sirocky, M. M., Ivezić, Ž., \& Elitzur, M. 2008, ApJ, 685, 147

Sirocky, M. M., Levenson, N. A., Elitzur, M., Spoon, H. W. W., \& Armus, L. 2008, ApJ, 678, 729

Sanders, D. B., Soifer, B. T., Elias, J. H., Madore, B. F., Matthews, K., Neugebauer, G., \& Scoville, N. Z. 1988, ApJ, 325, 74

Stern, D., et al. 2005, ApJ, 631, 163

Stern, D., et al. 2012, ApJ, 753, 30

Wilkes, B. J., et al. 2013, ApJ, 773, 15

Xue, Y. Q., et al. 2011, ApJS, 195, 10 